Applied Physics Letters, October 2007, Volume 91, Issue 18, Pages 183510-183510-3

\title{
In-plane excitation of thin silicon cantilevers using piezoelectric thin films
}

Glenn J T Leighton, Paul B Kirby

Department of Materials, Cranfield University, MK43 OAL, United Kingdom

Colin H J Fox

School of Mechanical, Materials and Manufacturing Engineering, University of Nottingham, Nottingham NG7 2RD, United Kingdom

This paper deals with the actuation of in-plane and out-of-plane motion of silicon cantilevers, using a single thin-film of lead zirconate titanate with a divided electrode configuration. Inplane actuation is demonstrated practically, and excellent agreement is obtained between theoretically predicted and experimentally measured resonant amplitudes, for the fundamental out-of-plane and in-plane modes of vibration of the fabricated test cantilevers. 
Many actuation principles have been applied in micro electro mechanical systems (MEMS) including thermal, magnetic, piezoelectric and electrostatic. Electrostatic actuation is most common because the fabrication processes are available at most MEMS facilities, but it has limitations that might be avoided by using piezoelectric actuation. Often electrostatic MEMS devices need high actuation voltages to obtain significant forces, leading to a requirement for additional circuitry when used with complementary metal oxide semiconductors, for which supply voltages are falling below $3 \mathrm{~V}$. In contrast, a micro-switch with thin-film piezoelectric actuation operating at $3 \mathrm{~V}^{1}$ has recently been demonstrated. Electrostatic actuation is capable of exciting both in-plane and out-of-plane motion depending on capacitor plate configuration, but separate capacitors are required for each direction. The comb-drive ${ }^{2}$ is an established means of increasing the available actuation force, but only at the expense of wafer area. Further disadvantages are the need to accurately maintain small electrode gaps and the snapdown effect which, in practice, typically limits the motion to less than one third of the electrode gap.

The most common configuration for piezoelectric MEMS structures is a piezoelectric thin film, with top and bottom electrodes deposited on a substrate structure. Application of a voltage across the piezoelectric film causes in-plane stresses via $\mathrm{d}_{31}$ action, which cause outof-plane displacement of the structure. The material with the highest electro-active coefficients that can be readily deposited in thin film form on silicon ( $\mathrm{Si}$ ) wafers is lead zirconate titanate (PZT). There have been numerous reports of the fabrication of unimorph MEMS structures using thin film PZT, particularly of Si cantilevers in which thicknesses of the Si and PZT layers are similar, and of the characterization of the out-of-plane bending that occurs when a voltage is applied across the $\mathrm{PZT} \mathrm{T}^{3,4}$. Under an alternating voltage significant 
displacements can be achieved using mechanical resonance of the structure in an out-of-plane mode of vibration, which has been used for applications including microwave filters and mass change in chemical sensing.

A recent paper ${ }^{5}$ presented a theoretical analysis of the mechanics of MEMS beam structures in which in-plane actuation is achieved using a piezoelectric film on the surface of the wafer with differentially excited electrode areas. In-plane actuation could have significant potential in applications such as microwave filters and inertial sensors. In this paper we demonstrate inplane motion excited in a MEMS device by a thin PZT layer, and make comparisons with theoretical predictions based on the analysis of Chen et $a l^{5}$.

The cantilevers used in this study were fabricated from silicon on insulator wafers with the PZT layer deposited on the Si device layer. The fabrication process has been previously described ${ }^{6}$ and requires a 200nm layer of silicon oxide $\left(\mathrm{SiO}_{2}\right)$ beneath the lower electrode. The PZT thin films were produced using sol gel deposition to give a film thickness of $1 \mu \mathrm{m}$. Platinum (Pt) was used as the bottom electrode for the growth of $\mathrm{Pb}_{1.1}\left(\mathrm{Zr}_{0.3}, \mathrm{Ti}_{0.7}\right) \mathrm{O}_{3}$. The choice of dimensions was based on the desired operating frequency $(90 \mathrm{kHz}$ out-of-plane and $600 \mathrm{kHz}$ in-plane), which to some extent was limited by available wafer thickness and processing capability. Figure 1 shows a fabricated cantilever which is the basis of the results presented in this paper and figure 2 shows a schematic cross section. The top-surface electrode is divided into two symmetrical strips of width $h$. In-plane actuation is achieved by applying equal-amplitude, anti-phase sinusoidal drive voltages to the two electrodes, causing alternating anti-phase contraction and expansion of the PZT on either side of the centerline of the cantilever. Out-of-plane excitation can be achieved with the same electrode layout by 
applying equal-amplitude, in-phase sinusoidal drive voltages, thus causing alternating, inphase expansion and contraction of the upper surface of the cantilever.

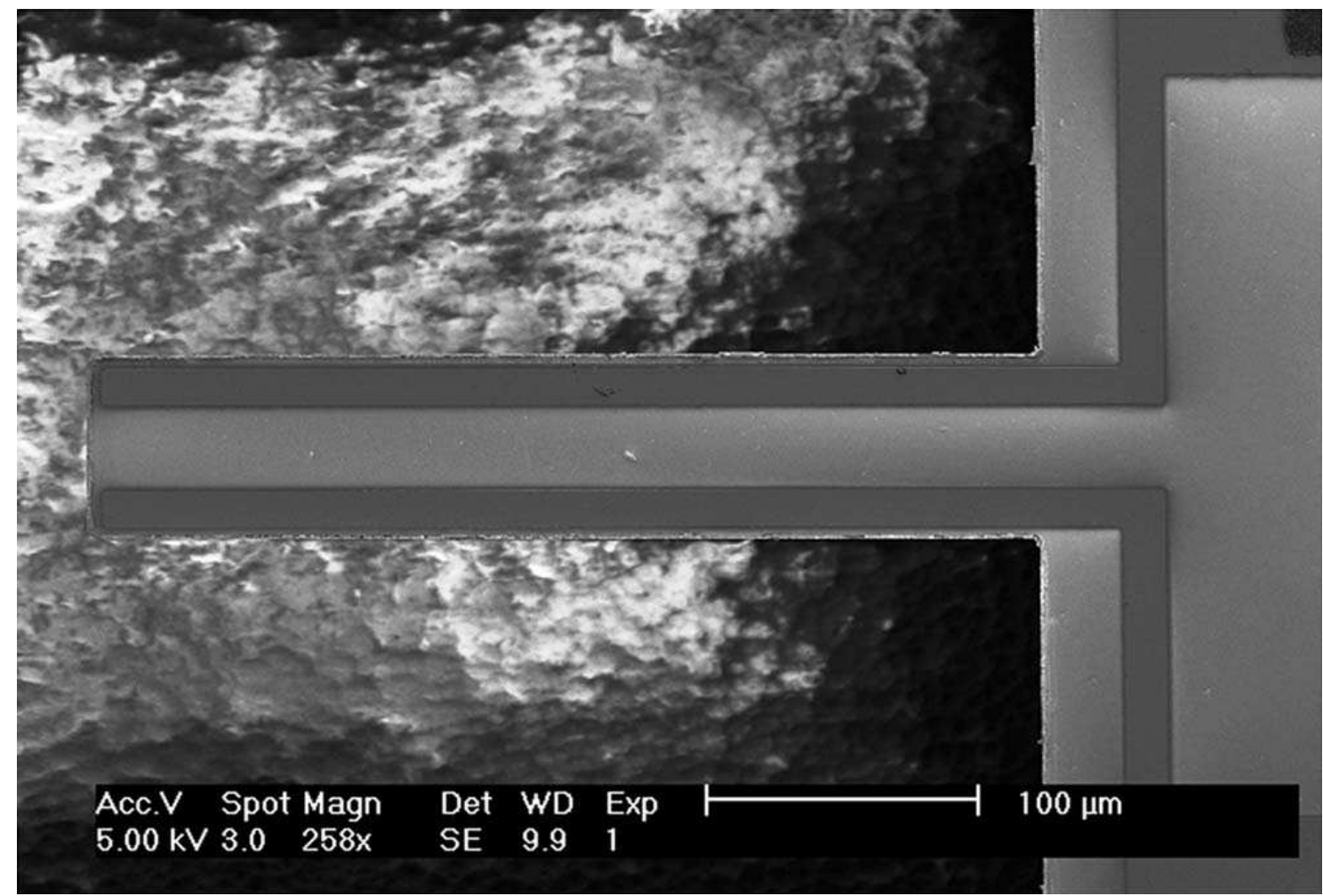

Figure 1. SEM image of cantilever specimen

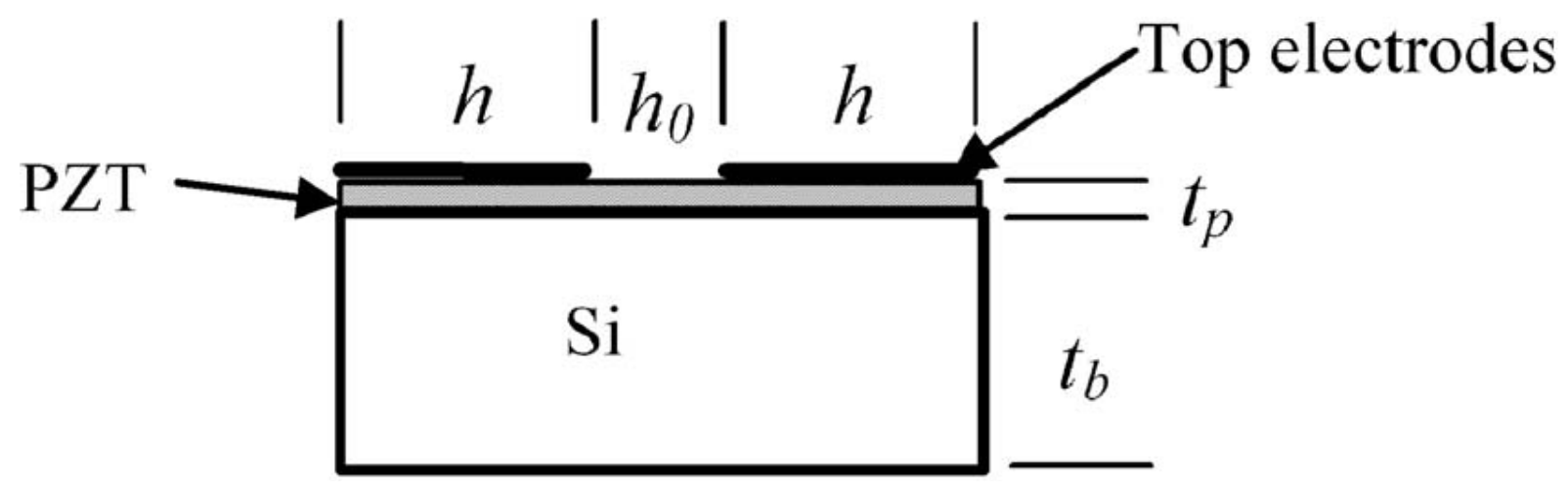

Figure 2. Schematic cross section of cantilever

The cantilevers had length $L=350 \mu \mathrm{m}$, width $\left(2 h+h_{0}\right)=75 \mu \mathrm{m}$ and thickness $t_{b}=10 \mu \mathrm{m}$ and the PZT thickness was $t_{p}=1 \mu \mathrm{m}$. The top electrode width was $h=20 \mu \mathrm{m}$ and the Pt thickness was 100nm. The material properties used in the calculations and finite element modeling are 
reported in ${ }^{4}$. X-ray characterization revealed PZT that was highly $<111>$ oriented in the perovskite phase with no evidence of pyrochlore. Additional evidence for complete conversion to the perovskite phase is that a dielectric constant and loss of 380 and 0.01 were measured, values which for films of this composition show high piezoelectric activity. The relevant $d_{31}$ value is given later.

The resonant amplitudes under in-plane and out-of-plane excitation were predicted using theoretical results from ${ }^{6}$, based on the dimensions defined in figure 2 . The in-plane actuation moment at cantilever tip due to anti-phased actuation of the electrodes can be expressed as

$$
M_{i-p}=\frac{E_{p} t_{p} \varepsilon_{0} h\left[h+h_{0}\right]}{1+\frac{E_{p} t_{p}}{12 E_{b} I_{b}}\left[\left(h_{0}+2 h\right)^{3}-h_{0}^{3}\right]}
$$

where $\varepsilon_{O}=d_{31} V / t_{p}$ is the free piezoelectric strain and $I_{b}=t_{b}\left(h_{0}+2 h\right)^{3} / 12 . V$ is the actuation voltage, $E_{b}$ and $E_{p}$ are the Young's moduli of the substrate Si and PZT respectively.

When the electrodes are excited by equal voltages of the same polarity, the effective out-ofplane actuation bending moment can be expressed as

$$
M_{o-p}=\frac{\varepsilon_{0}}{\frac{1}{E_{b} A_{b}}+\frac{t_{b}\left(t_{b}+t_{p}\right)}{4 E_{b} I_{b}^{\prime}}+\frac{1}{E_{p} A_{p}}} \frac{t_{b}}{2}
$$

where $A_{\mathrm{b}}, A_{\mathrm{p}}$ are the cross sectional areas of the substrate and piezoelectric material. 
Using a modal expansion approach ${ }^{7}$ in which only the fundamental bending mode is retained, the displacement amplitude at the cantilever tip under harmonic excitation at the resonant frequency, $\omega_{S}$, of the relevant mode of vibration can be expressed as:

$$
y_{R E S}(L)=\frac{M \phi^{\prime}(L)}{\rho A L} \frac{Q_{S}}{\omega_{S}^{2}} \phi(L)
$$

where $M$ is the relevant excitation moment given by equation (1) or (2), $L$ and $\rho A$ are the length and effective mass per unit length and $Q_{S}$ is the mechanical quality factor of vibration mode $s$, to be determined experimentally. $\phi(L)$ and $\phi^{\prime}(L)$ are the displacement and slope of the relevant Eigen function ${ }^{8}$, evaluated at the tip of the cantilever $(x=L) . I_{b}^{\prime}=\left(h_{0}+2 h\right) t_{b}^{3} / 12$. Equations (1) and (2) neglect the stiffening effects of the $\mathrm{SiO}_{2}$ and $\mathrm{Pt}$ layers. This is

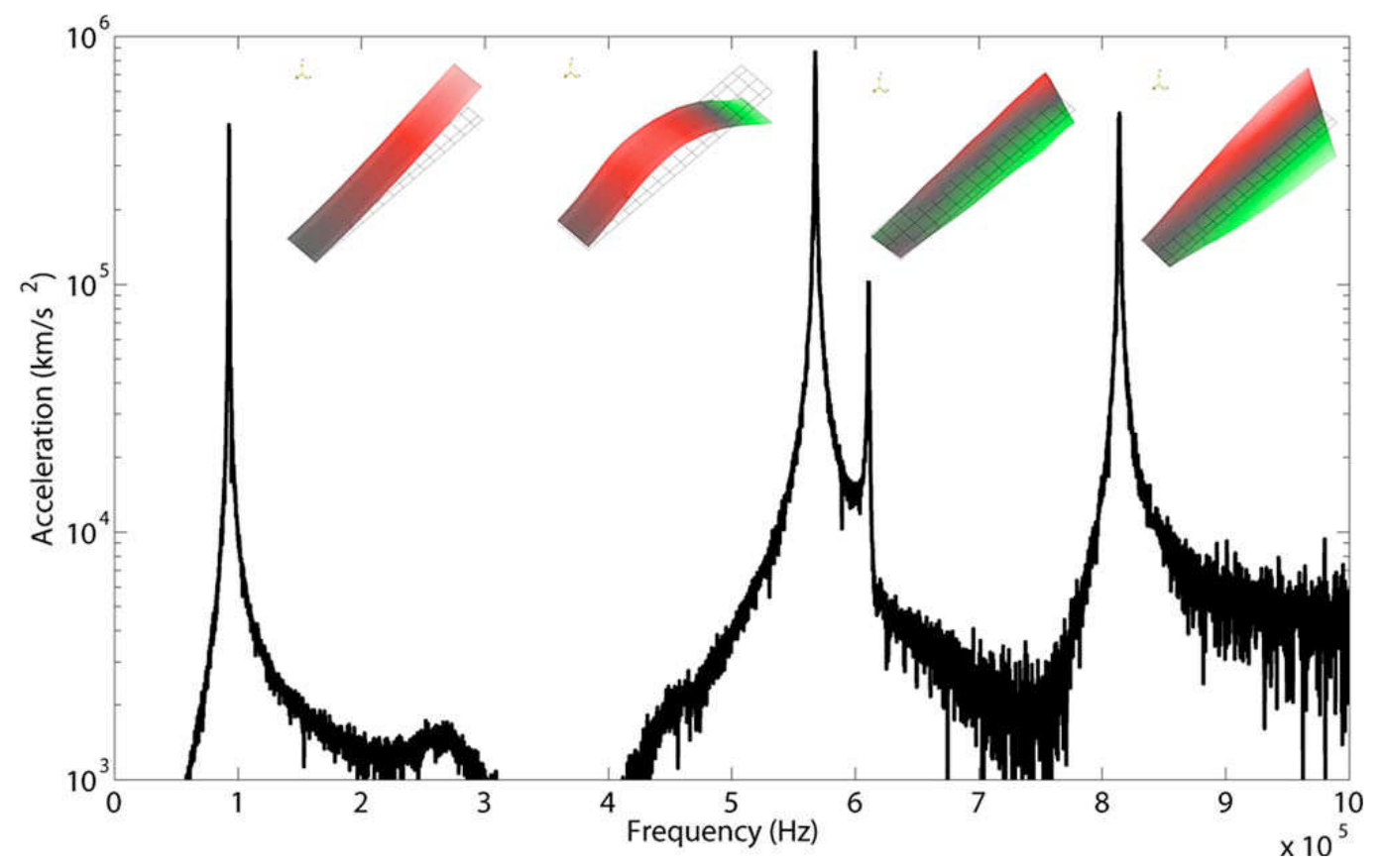

Figure. 3. (Color online) Frequency response of cantilever specimen. _Inset images show mode shapes of the first, second, and fourth modes, and the out-of-plane displacement of the third mode).

reasonable in view of their small thickness and because the elastic moduli are of the same order as that of $\mathrm{Si}$. The mass of the electrodes is significant due to the high density of $\mathrm{Pt}$ 
$\left(21090 \mathrm{~kg} / \mathrm{m}^{3}\right)$ compared to that of $\mathrm{Si}\left(2330 \mathrm{~kg} / \mathrm{m}^{3}\right)$ and this is accounted for in the effective $\rho A$ used in equation (3). The density, and hence the contribution to $\rho A$, of the $\mathrm{SiO}_{2}$ is very similar to that of $\mathrm{Si}$.

For the experimental measurements, an alternating excitation of $3 \mathrm{~V}$ was applied to the electrodes, phased appropriately as described earlier. The out-of-plane modal response and mode shapes were measured using the micro-scanning laser vibrometer (MSV) of the Polytech micro motion analyzer (MMA) 300. Figure 3 shows a frequency response measurement made using anti-phase excitation voltages. For presentational purposes the acceleration response has been plotted with a logarithmic scale, which increases the prominence of the small amplitude peaks. The response has prominent peaks corresponding to resonance of the first two out-of-plane bending modes at $90 \mathrm{kHz}$ and $558 \mathrm{kHz}$ and the first twisting mode at $824 \mathrm{kHz}$. The inset images in figure 3 show the corresponding measured mode shapes. A smaller peak is visible at $610 \mathrm{kHz}$, which is close to the predicted frequency of the fundamental in-plane bending mode of vibration.

The natural frequencies of the observed modes were within $\sim 5 \%$ of theoretical predictions based on textbook analyses ${ }^{8}$ for uniform beams and within $1 \%$ of detailed Finite Element predictions, both of which included the Pt electrodes, giving confidence that the actual test structures conformed to the design.

The fact that the $90 \mathrm{kHz}$ and $558 \mathrm{kHz}$ in-plane modes were excited by the nominally antisymmetric excitation (i.e. anti-phase voltages applied to the electrodes) indicates the presence of slight deviations of the actual structure from the symmetric ideal. This could be due, 
amongst other things, to slight non-uniformity in the PZT film thickness and properties or small etching imperfections affecting the accuracy of dimensions and electrode position. The fact that the resonant peaks at $90 \mathrm{kHz}, 558 \mathrm{kHz}$ and $824 \mathrm{kHz}$ are the most prominent in Figure 3 is because the laser vibrometer was being used in a mode that detects out-of-plane motion. The appearance of the smaller $610 \mathrm{kHz}$ peak corresponding to an in-plane mode is again attributable to small asymmetries in the cantilever structure, which mean that the nominally pure in-plane mode actually contains an out-of-plane displacement component.

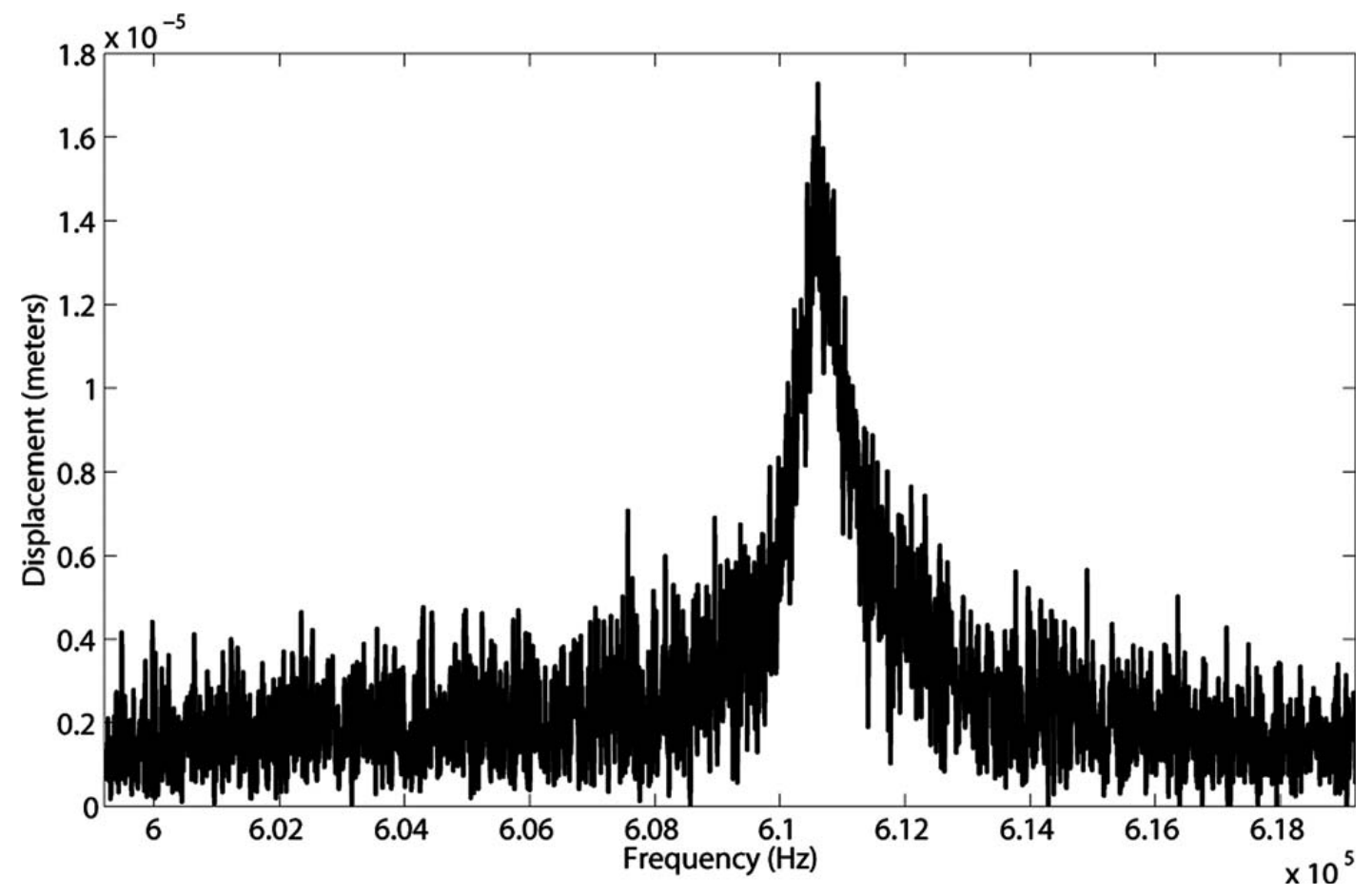

Figure 4. Frequency response of in-plane mode with description of $Q$ determination

Using anti-phase excitation voltages, the resonance of the $610 \mathrm{kHz}$ in-plane mode was studied in detail over a $20 \mathrm{kHz}$ frequency range around $610 \mathrm{kHz}$. In-plane displacements were measured using the planar motion analyzer capability of the MMA 300. This acquires a sequence of images at different phase angles over the vibration cycle using a light emitting diode to provide strobed illumination of the sample. The images were then processed to 
determine the motion with an accuracy of $\sim 4 \mathrm{~nm}$ at $50 \mathrm{x}$ magnification. The resulting frequency response is shown in Figure 4, showing a maximum tip displacement of $17.2 \mu \mathrm{m}$. This clearly indicates the actuation of significant in-plane displacement of the cantilever using a single thin film of piezoelectric material deposited on the surface of the wafer, with appropriate electrode arrangement and anti-phased excitation.

The mechanical $Q$-factors for the $610 \mathrm{kHz}$ in-plane mode was estimated using the half-power bandwidth of the frequency response curve in Figure 4 where the centre frequency is 610.6 $\mathrm{kHz}$ and the bandwidth is $0.18 \mathrm{kHz}$, giving $Q_{610}=3392$. From a similar test using $3 \mathrm{~V}$ inphase excitation to excite the $90 \mathrm{kHz}$ out-of-plane mode it was found that the resonant displacement amplitude at the tip was $4.7 \mu \mathrm{m}$ and that $Q_{90}=75$.

The $\mathrm{d}_{31}$ piezoelectric coefficient was determined experimentally using the MSV as described in ${ }^{4}$ and it was found that $d_{31}=22 \times 10^{-12} \mathrm{~m} / \mathrm{V}$. Based on the nominal specimen dimensions, the measured values of $Q, d_{31}$ and natural frequency $\omega_{S}$, and assuming $3 \mathrm{~V}$ excitation, equations (1) - (3) were used to predict the resonant amplitudes at the cantilever tip for the $90 \mathrm{kHz}$ (outof-plane) and $610 \mathrm{kHz}$ (in-plane) modes. Table 1 shows the predicted and measured values. For the out-of-plane mode there is excellent agreement (within 2.2\%) between the measured and predicted response amplitudes. For the in-plane mode the agreement is less good but is still within $20 \%$. The previously mentioned effects of slight warping or imperfection in the structure and the relative difficulty in measuring in-plane displacements as compared to outof-plane displacements are factors that contribute to the reduced level of agreement. 
Table I - Comparison of measured and theoretical resonant response amplitudes

\begin{tabular}{lll}
\hline \hline & $\begin{array}{l}\text { Measured } \\
\text { amplitude } \\
(\mu \mathrm{m})\end{array}$ & $\begin{array}{l}\text { Predicted amplitude } \\
(\text { Eqn }(1)-(3)) \\
(\mu \mathrm{m})\end{array}$ \\
\hline $\begin{array}{l}\text { In-plane mode } \\
\text { at } 610 \mathrm{kHz}\end{array}$ & 17.2 & $20.5^{(\mathrm{a})}$ \\
$\begin{array}{l}\text { Out-of-plane } \\
\text { mode at } 90 \mathrm{kHz}\end{array}$ & 4.7 & $4.8^{(\mathrm{b})}$ \\
\end{tabular}

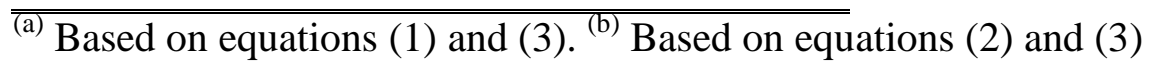

Out-of-plane modes have been demonstrated by many workers and equation (3) can be regarded as a well established theoretical result. The level of agreement for the out-of-plane mode therefore gives confidence in the measured parameters as well as the validity of equation (2). For the in-plane mode, in addition to the practical demonstration of strong actuation, the level of agreement between measured and predicted amplitudes gives confidence in the validity of the previously untested equation (1) as a useful tool for design calculations.

The authors acknowledge the financial support of the European Union through European Projects 027926 "Q2M" and 507352 “AMICOM".

${ }^{1}$ H.-C. Lee, J.-Y. Park and J.-U. Bu, IEEE Microw. Wirel. Compon. Lett. 15, 202 (2005).

${ }^{2}$ W. C. Tang, T. H. Nguyen and R. T. Howe, IEEE Micro Electro Mech. Syst. 53 (1989)

${ }^{3}$ P. B. Kirby, R.V. Wright, P. Gaucher, P. Galtier, L. Kofoed, J. O. Gullov, W. Von Munch, D.

Eichner, B. Ploss and J. K. Kruger, J. Phys. IV. 8, 161 (1998).

${ }^{4}$ Z. Huang, G. Leighton, R. Wright, F. Duval, H. C. Chung, P. Kirby and R. W. Whatmore, Sens. Actuator A-Phys. 135, 660 (2007). 
${ }^{5}$ X. Chen, C. H. J. Fox and S. McWilliam, Proc. SPIE 5049, 371 (2003).

${ }^{6}$ H. W. Jiang, P. Kirby and Q. Zhang, Proc. SPIE 4979, 165 (2003).

${ }^{7}$ G. B. Warburton, The Dynamical Behaviour of Structures $2^{\text {nd }}$ Ed (Pergamon International, 1976).

${ }^{8}$ R. D. Blevins, Formulas for natural frequency and mode shape (Krieger Publishing company, 1979). 
Figure 1 SEM image of cantilever specimen

Figure 2 Schematic cross section of cantilever

Figure 3 Frequency response of cantilever specimen.

(Inset images show mode shapes of the $1^{\text {st }}, 2^{\text {nd }}$ and $4^{\text {th }}$ modes, and the out of plane displacement of the $3^{\text {rd }}$ mode)

Figure 4 Frequency response of in-plane mode with description of Q determination 